\title{
Characterizing macromolecular samples using SAXS and WAXS
}

\author{
Angela Criswella ${ }^{a}$ Mark Del Campo ${ }^{b}$, Thom Hendrixson ${ }^{c}$ \\ Rigaku Americas Corp., 9009 New Trails Dr., The Woodlands, TX 77381, angela.criswell@rigaku.com \\ Rigaku Americas Corp., 9009 New Trails Dr., The Woodlands, TX 77381, mark.delcampoo@rigaku.com \\ Rigaku Americas Corp., 9009 New Trails Dr., The Woodlands, TX 77381, thom.hendrixson@rigaku.com
}

Small angle X-ray scattering (SAXS) is a useful technique for extracting structural information from biological samples in solution. Most biological SAXS samples are aqueous solutions with proteins present in low concentration. In other cases, it may be of interest to study macromolecules at high concentration, at high viscosity or at conditions in which macromolecules are expected to crystallize. In such cases, SAXS can provide useful information about the inherent structure and phase of macromolecules.

Rigaku Oxford Diffraction's SAXS platform for biological SAXS, the BioSAXS-2000 nano system, is well suited for analysis of all types of biological samples, independent of concentration, viscosity and phase. This compact system includes unique features, including modularly designed sample stages, enabling quick sample stage changes for those labs interested in studying materials or anisotropic systems.

Additionally, wide-angle X-ray scattering (WAXS) capabilities are optional and offer a high flux option for WAXS data collection. In this studying, we describe the versatility offered by the new BioSAXS-2000 nano system for biological SAXS. 